\title{
Optimum Practical Design of Distributed and Asynchronous Power Control for Wireless Networks with Shared Bands
}

\author{
Stepan KUCERA $^{\dagger \text { a) }}$, Student Member, Koji YAMAMOTO ${ }^{\dagger}$, Member, and Susumu YOSHIDA $^{\dagger}$, Fellow
}

\begin{abstract}
SUMMARY The present paper proposes two novel and practical schemes for distributed and asynchronous power control in wireless ad hoc networks, in which users dynamically share several frequency bands as in "cognitive radio" networks. These schemes iteratively adjust transmit powers of individual network transmitters with respect to mutually caused interference in the shared bands. Their most attractive feature is that they find network-wide acceptable trade-offs to diverse signal-to-noise and interference (SINR) requirements and efficiently use techniques of stochastic approximation and time-averaging to guarantee a robust performance in random channels. Advantageously, both proposed algorithms do not assume any particular modulation, coding, QoS measure definition or network architecture, which assures their high applicability in the industry and research. Moreover, the broad definition and non-linear nature of these schemes mathematically generalize and thus encompass as a special case many widely deployed power control schemes such as e.g. those for achieving fixed SINR targets or using game-theoretic utility maximization. Simulations are provided to illustrate our approach and its better performance compared to standard algorithms.

key words: cognitive radio, ad hoc networks, power control, distributed, asynchronous, equilibrium, frequency band sharing
\end{abstract}

\section{Introduction}

Adaptive transmit power allocation and control is a challenging problem in the design of mobile wireless networks with inter-user interference, because an effective power and interference management is essential to promote the quality of service and communication efficiency [1].

An intensive research in the context of the third generation $(3 \mathrm{G})$ and beyond systems such as e.g. the UMTS, cdma2000 or WCMDA cellular data networks [2] has lead to the development and deployment of several wellperforming power control schemes. They commonly consist of two or even more nested transmit power control loops, whose purpose is to maintain the received uplink signal-tointerference ratio (SINR) at some target level throughout the transmission [2]. This in turn allows to guarantee certain quality-of-service $(\mathrm{QoS})$ in terms of constant bit-error rate, outage probability or transmission efficiency [3]. Theoretically, such schemes can be successfully approximated by models, in which transmit power control is assigned as a linear function of the receiver interference, whose slope corresponds to the target SINR [4], [5].

Manuscript received October 26, 2006.

Manuscript revised January 16, 2007.

Final manuscript received March 9, 2007.

${ }^{\dagger}$ The authors are with the Department of Communications and Computer Engineering, Graduate School of Informatics, Kyoto University, Kyoto-shi, 606-8501 Japan.

a) E-mail: kucera@hanase.kuee.kyoto-u.ac.jp

DOI: 10.1093/ietfec/e90-a.7.1261
Recent advances in wireless engineering have also witnessed the emergence of two new approaches to system design of wireless networks. First, it is the concept of " $\operatorname{cog}$ nitive radio" [6], in which cognitive terminals search for available frequency bands and use them for temporary transmissions as secondary users instead of idle primary owners. The other approach is based on multihop "ad hoc" networking [7], which is carried out without the support from any predetermined (cellular) infrastructure.

Such networks are attractive conceptually and in applications, but their power control implies new problems such as the need for QoS-aware distributed and asynchronous solutions, which is in strong contrast to the centralized and linear power control of above mentioned cellular systems. To answer the new challenge and avoid also the possible divergence of the early algorithms [4], [5], recent works inspired e.g. by [8]-[11] have tried to apply game-theoretic maximization of QoS related utility functions. These works, however, depart from strongly application dependent system models (cellular CMDA system) and offer results with low mathematical generality or practical universality (assuming strong restrictions on used utility functions). Moreover, we showed in our previous work [12] several practical and mathematical limitations of the utility emphasizing power control models.

\section{Paper Contributions and Outline}

Developing our own innovative theoretical results towards real-life applications and building upon its advanced mathematical tools [13]-[15], we describe in a detailed fashion two new ways of how to practically equip wireless terminals with the ability of asynchronous and distributed power control in the attractive context of modern cognitive networking with no (cellular) infrastructure support.

For this purpose, we avoid in accordance with [12] the limited utility maximization approach and apply our own more flexible best-response approach [14] to distributed power control in shared bands. Therein, each active transmitter periodically updates its transmit power to a value, which is given by a best-response function having the cochannel interference at the receiver as its input.

Importantly, we leave upon network users themselves to distributively define such functions according to their individual preferences and local network conditions. Therefore, said functions will be discussed as time-variant and generally nonlinear without any further assumptions, which 
in fact generalizes the purely linear case of [4], [5], resp. 3G systems with fixed SINR targets and also the one of [8]-[11] with their stationary and simplified cellular models. This allows to analyze even highly complicated systems with nonlinear power allocation strategies and SINR preferences variable in time and space such as e.g. in systems, in which time instants with low interference are used for bursty data transmissions at high SINR, whereas during stronger interference conditions the transmitters prefer to rather wait and save power.

Noting that lack of similarly general, yet complex and unified references in this field, we furthermore state that the two proposed power control schemes are characterized by the following innovative and non-trivial features:

- distributed and asynchronous convergence to stable solutions to the trade-off between link-centric transmit power allocation preferences and resulting interference to others in used shared bands by iteratively solving a non-cooperative power control/allocation game;

- achieving finally an overall acceptable and steady equilibrium state, in which each transmitter's power is optimally adapted to the equilibrium interference from others in the sense of eliminating any rational incentive to deviate therefrom;

- robust performance in random channels of both ergodic and non-ergodic type thanks to the usage of stochastic approximation and time-averaging.

The paper is organized as follows. Section 3 summarizes the two power control schemes for both ergodic and non-ergodic channels. In Sects. 4 and 5, we describe necessary design techniques for assuring their convergence to overall acceptable network-wide equilibria. Section 6 discusses an illustrative design example, characterized by global exponential convergence to a unique equilibrium. Section 7 presents numerical results, proving the potential of our approach to outperform standard game-theoretic algorithms. Conclusion follows in Sect. 8.

\section{System Model and Summary of Proposed Schemes}

In our model, we assume a mobile multihop ad hoc network with several mutually orthogonal (noninteracting) frequency bands and composed of cognitive terminals (see Fig. 1). These scan the usable frequency spectrum and communicate opportunistically in bands, which are currently not used by their primary owners. The scanning and band selection process can be based for example on the evaluation of current interference constraints as in [6]. Clearly, each frequency band can accommodate several simultaneously active links. Links correspond to concurrent single-hop transmissions of primary and/or secondary users.

The orthogonality among different channels can be achieved by combining techniques of time-division multiple access (TDMA) or frequency-division multiple access (FDMA). Within one orthogonal channel, different links can use code-division multiple access technique (CDMA) for

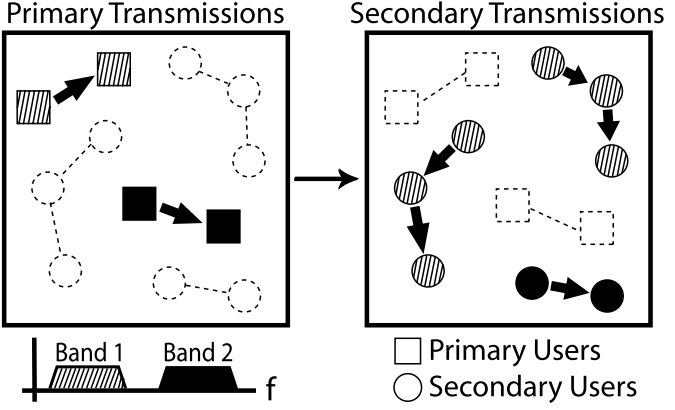

Fig. 1 Schematic illustration of sharing of available frequency bands, freed temporarily by their licensed primary owners, by unlicensed secondary users.

separating ongoing transmissions, which however does not eliminate the necessity of power control due to the near-far effect and others [16].

In order to propose our two schemes, suppose in general $N$ simultaneously active links in one given band and let channel gain $h_{i j}$ represent the power loss due to the channel from the transmitter of link $i$ to the receiver of link $j$ for $i, j \in \mathscr{I}=\{1, \ldots, N\}$. This comprises path loss, multipath fading, shadowing and other radio propagation effects, as well as CDMA processing gain [1].

We think of the power allocation/control process as of individual decision making in a non-cooperative environment. At the beginning of a data transmission period, the $i$ th link's transmit power $\sigma_{i}$ - used for transmitting the data signal - is set to some initial value $\sigma_{i}^{\text {init }}$ (later conditions for global convergence are discussed, whereby in such a case any $\sigma_{i}^{\text {init }}$ is possible).

During the subsequent transmission time, each link's transmitter adjusts its power $\sigma_{i}$ periodically with period $T>$ 0 in time instants $t_{i}^{k} \in\left[\tau^{k}, \tau^{k+1}\right.$ ) for all $\tau^{k}$ and integer $k$ such that $\tau^{0}=0$ and $\tau^{k+1}-\tau^{k}=T$. Transmit power updates are assumed to be asynchronous, i.e., update times $t_{i}^{k} \neq t_{j}^{k}$ for each links $i$ and $j \neq i$ and given $k$.

To be more specific, the power updates are carried out in the following way. During each update period $\left[\tau^{k}, \tau^{k+1}\right)$, every link's receiver measures the power, at which it receives data packets from its transmitter, and also measures the interference power from other links. For example, the interference power can be measured in short time instances located inbetween the intervals, dedicated to the actual reception of individual data packets.

In the next step, the receiver calculates the most desirable received power of the data packets signal using socalled best-response function $B_{i}$ [13], whose input is the measured co-channel interference power. This function assigns to each possible co-channel interference value the most desirable received power necessary to overcome the given interference from other links and achieve an acceptable connection according to link $i$ 's individual preferences. Having calculated the desired received power, the receiver calculates an update factor $f$, given by the ratio of the calculated desired received power and the value of the mea- 
sured actual received power, and submits this factor to the transmitter in a short signaling packet (or for example jointly with the acknowledgement of a data block reception). The link's transmitter then updates its transmit power to a new power, obtained by multiplication of the previously used power output with the acquired factor $f$.

Throughout this work, we assume $B_{i}$ to be generally different (and possibly slowly time-variant) among individual links in order to respect their different network conditions and preferences on SINR. Based on this general system model, we propose the following two asynchronous and distributed power control schemes for each individual link $i$ : (a) a scheme, suitable for ergodic channels; and (b) a scheme, suitable for non-ergodic channels.

\subsection{Power Control for Ergodic Channels}

A channel gain process is ergodic, if roughly speaking a short time scale average of $h_{i j}$ is equal to its overall average. This means that the randomness of the channel gain can be averaged out (removed) over time. For this reason long-term constant bit rates can be supported in e.g. AWGN channels or fast Rayleigh fading channels. Therefore, in the proposed power control scheme for ergodic channels, each link's receiver estimates its average interference power by simply averaging several interference measurements during each update period and based on this averaged information calculates the corresponding best-response transmit power using its $B_{i}$. Then it notifies the transmitter about the update factor $f$, which updates its power by multiplication with this factor. More precisely, said scheme is given by the following protocol (see Fig. 2 for a schematic illustration)

1. in each time period $\left[t_{i}^{k}, t_{i}^{k+1}\right)$, link $i$ 's receiver (RX)

- collects over the period $\left[t_{i}^{k}, t_{i}^{k+1}\right)$ samples of the received power $\sigma_{i}^{\mathrm{RX}}=h_{i i} \sigma_{i}$ from its transmitter and also the interference power $I_{i}^{\mathrm{RX}}=\boldsymbol{h}_{-i}^{\mathrm{T}} \boldsymbol{\sigma}_{-i}$ from other links than link $i$ (index $-i$ ), where vectors

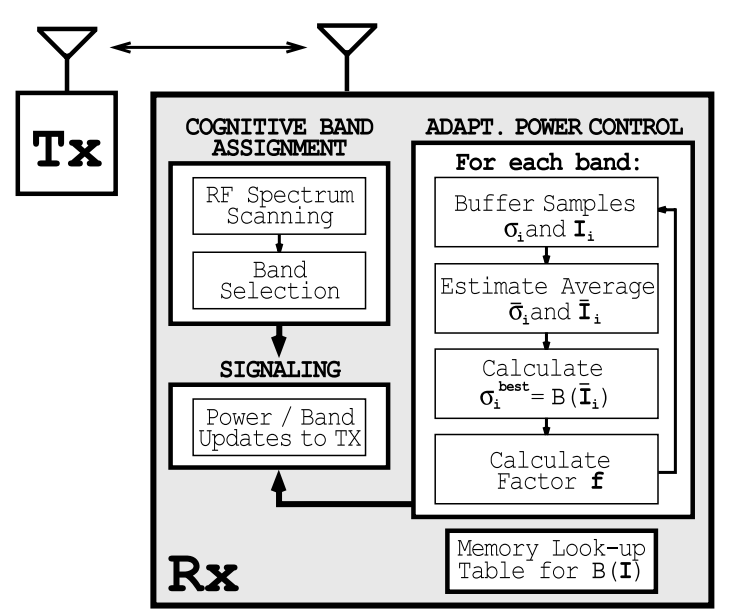

Fig. 2 Schematic representation of the proposed distributed power control scheme for cognitive ad hoc networks with ergodic channels using general best-response functions $B_{i}$ (index $R X$ is omitted for clarity). $\boldsymbol{h}_{-i}$ and $\boldsymbol{\sigma}_{-i}$ have elements $h_{i, j \neq i}$, resp. $\sigma_{j \neq i}$;

- estimates based on these samples the corresponding average values $\bar{\sigma}_{i}^{\mathrm{RX}}$ and $\bar{I}_{i}^{\mathrm{RX}}$ of the received power, resp. interference power from other links, sharing the same band;

- calculates the most desirable received power (or "best response") $\sigma_{i}^{\mathrm{RX} \text { best }}=B_{i}\left(\bar{I}_{i}^{\mathrm{RX}}\right)$ for the given average interference value $I_{i}^{\mathrm{RX}}$

- submits the factor $f=\sigma_{i}^{\mathrm{RX}}{ }_{\text {best }} / \bar{\sigma}_{i}^{\mathrm{RX}}$ to its transmitter (TX) e.g. in a data acknowledgement packet or separately over a dedicated signaling channel;

2. said transmitter of link $i$ updates upon the acquisition of the factor $f$ its transmit power $\sigma_{i}$ by multiplication with $f$ to a new output power $\sigma_{i}^{k+1}=f \sigma_{i}^{k}$,

whereby $B_{i}$ are discussed as functions with a general form in order to incorporated different needs and requirements of different industrial designers. In principle, such a power control scheme is attractive from a practical point of view, because $B_{i}$ can be easily implemented in any device by a simple memory chip, containing a lookup table with values of $B_{i}$ for different interference values (see Fig. 2).

\subsection{Power Control for Non-ergodic Channels}

Channel gain $h_{i j}$ is non-ergodic, if its short time scale average is not equal to the overall average. This unfortunately means that long-term constant bit rates cannot be theoretically supported by simply averaging out the channel gain randomness over time as in the previous case. A more advanced design of power control is therefore needed. For this purpose, we propose the following stochastic approximation-based algorithm. In every update period, each link's receiver measures similarly to the previous cases the average values of interference and received power, but calculates its power update with respect to $B_{i}$ as equal to $\bar{\sigma}_{i}^{\mathrm{RX}}+\alpha_{i}^{k}\left(B_{i}\left(\bar{I}_{i}^{\mathrm{RX}}\right)-\bar{\sigma}_{i}^{\mathrm{RX}}\right)$, where $\alpha_{i}^{k}$ is the algorithmic stepsize, slowly decreasing with increasing $k$. If the power update exceeds the maximum power limit $P_{i}^{\max }$, it is truncated to some restart value $\sigma_{i}^{\text {init }}<P_{i}^{\max } \forall i \in \mathscr{I}$. Then similarly to the previous case, the receiver notifies the transmitter about the update factor $f$, which then updates its transmit power output for data by multiplication with this factor. More precisely, we define the non-ergodic channel algorithm as follows (see Fig. 3 for a schematic illustration):

1. in each time period $\left[t_{i}^{k}, t_{i}^{k+1}\right)$, link $i$ 's receiver

- estimates similarly to the previous scheme the average values $\bar{\sigma}_{i}^{\mathrm{RX}}$ and $\bar{I}_{i}^{\mathrm{RX}}$ of its received power, resp. interference power from other links based on samples of $\sigma_{i}^{\mathrm{RX}}$ and $I_{i}^{\mathrm{RX}}$;

- calculates $\sigma_{i}^{\mathrm{RX} \text { best }}=\bar{\sigma}_{i}^{\mathrm{RX}}+\alpha_{i}^{k}\left(B_{i}\left(\bar{I}_{i}^{\mathrm{RX}}\right)-\bar{\sigma}_{i}^{\mathrm{RX}}\right)$, where $\alpha_{i}^{k}$ is the link $i$ 's own algorithmic stepsize such that (i) $a_{i}^{k}>0$; (ii) $a_{i}^{k} \stackrel{k \rightarrow \infty}{\longrightarrow} 0$; (iii) $\sum_{k=1}^{\infty} a_{i}^{k}=\infty$ and (iv) $0<\liminf _{k \rightarrow \infty} a_{i}^{k} / a_{j}^{k}=$ $\lim \sup _{k \rightarrow \infty} a_{i}^{k} / a_{j}^{k}$ a.s. $\forall j \neq i$. 


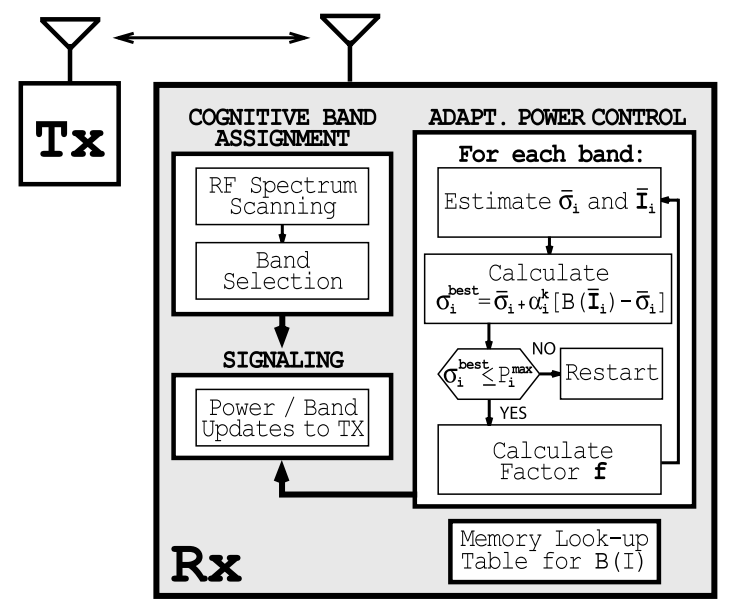

Fig. 3 Schematic representation of the proposed distributed power control scheme for cognitive ad hoc networks with non-ergodic channels using general best-response functions $B_{i}$ (index $R X$ is omitted for clarity).

- compares $\sigma_{i}^{\mathrm{RX} \text { best }}$ with its maximum power limit $P_{i}^{\max }>0$ and:

- if $\sigma_{i}^{\mathrm{RX} \text { best }}>P_{i}^{\max }$, then $\sigma_{i}^{\mathrm{RX}}$ best is set to a restart value $\sigma_{i}^{\text {init }}<P_{i}^{\max } \forall i$ (case "NO" in Fig. 3);

- if $\sigma_{i}^{\mathrm{RX}}$ best $\leq P_{i}^{\max }$, the algorithm proceeds to the next step (case "YES" in Fig. 3);

- submits the factor $f=\sigma_{i}^{\mathrm{RX}} \mathrm{D}_{\text {best }} / \bar{\sigma}_{i}^{\mathrm{RX}}$ to its transmitter e.g. in a data acknowledgement packet or separately over a dedicated signaling channel;

2. said transmitter of link $i$ updates upon acquisition of factor $f$ its transmit power $\sigma_{i}$ by multiplication with $f$ to a new output power $\sigma_{i}^{k+1}=f \sigma_{i}^{k}$,

whereby also in this case best-response functions $B_{i}$ are discussed in a general form in order to incorporated various design needs and networking requirements. Similarly, a physical implementation of $B_{i}$ by means of a simple memory chip with a lookup table can be used (see Fig. 3).

\subsection{Mathematical Model}

We have shown in [15] that distributed power control schemes such as the proposed ones can be for analytical purposes advantageously formulated as a strategic game [17] and in fact generalize all the previous game-theoretic schemes with QoS utility maximization [12]. This will be used in the following sections to analyze their properties. Setting $\beta_{i}\left(\boldsymbol{\sigma}_{-i}\right) \stackrel{\text { def }}{=} \frac{1}{h_{i i}} B_{i}\left(\boldsymbol{h}_{-i}^{\mathrm{T}} \boldsymbol{\sigma}_{-i}\right)$, the game is formally described by the following three elements:

1. a finite set of active links $i \in \mathscr{I}$ (game players), simultaneously accessing the shared medium;

2. a transmit power $\sigma_{i} \in \Sigma_{i}$ (game strategy) for each link $i$, where $\Sigma_{i} \subset \mathfrak{R}^{1}$ defines the power profile of link $i$;

3. link $i$ 's best-response function $\beta_{i}$ (transmit power update law); whereby $\boldsymbol{\Sigma}_{-i}$ with elements $\boldsymbol{\sigma}_{-i}$ represents the strategy profile of opponent links $-i$ and is given by Cartesian product of strategy profiles $\Pi^{\mathscr{I} \backslash\langle i\rangle} \boldsymbol{\Sigma}_{j}$ for all $j \in \mathscr{I}$ and $j \neq i$. The strategy profile $\Sigma$ with elements $\sigma$ of the entire game is given by $\Pi^{\mathscr{I}} \boldsymbol{\Sigma}_{i}$ for all $i \in \mathscr{I}$.

\section{Step 1: How to Assure Optimal Control Outcome}

Every designer of adaptive power control schemes needs to first assure by technical means the existence of a desirable power control outcome, to which the proposed schemes would preferably converge. Clearly, the most desirable outcome of a dynamic power control consists in a final steady state, in which each link $i$ 's choice of transmit power is optimally adapted to the interference from other links with respect to given $\beta_{i}$. In terms of our power control game model with given links as players, it is a steady state, from which none of concurrently transmitting links has an incentive to unilaterally deviate by changing its transmit power unless other active links change their power.

Evidently, if some link terminates its transmission or a new link suddenly starts to transmit, a new equilibrium has to be dynamically found by some additional iterations, reflecting the changed number of active links in the network and resulting different interference conditions. Therefore, each transmitter should use the proposed schemes during the entire transmission period in order to easily react to such events. The following design technique, valid for both proposed algorithm, introduces a way of how to technically assure the existence of such a desirable outcome in the form of Nash equilibrium [17]:

Design Technique 1 (Assuring Nash equilibrium existence by shaping $\beta_{i}$ ): Let each link's $i$ transmit power range $\Sigma_{i}$ be a continuous power range from zero powers to maximum power $P_{i}^{\max }$. Assume that best-response functions $\beta_{i}$ for all $i \in \mathscr{I}$ are implemented by a detailed memory look-up table, which emulates a continuous function, quantized with a very small quantization step $\Delta$. Then if the memory lookup table assigns to every interference value a power smaller than $P_{i}^{\max }$ and $\Delta \rightarrow 0$, then there always exists a Nash equilibrium in the distributed and asynchronous power control.

Proof: The notion of said steady state is formalized by a commonly used concept of Nash equilibrium [17], which we define accordingly to [14] as a set of allocated powers $\hat{\sigma}$ of individual links such that for each link $\hat{\sigma}_{i}=\beta_{i}\left(\hat{\boldsymbol{\sigma}}_{-i}\right)$.

Assume now a joint best-response function $\boldsymbol{\beta}$ as a Cartesian product of $\beta_{i}$ for all $i \in \mathscr{I}$. In order to work with $\boldsymbol{\beta}$ in the context of the entire strategy space $\boldsymbol{\Sigma}$, we formally substitute the argument $\sigma_{-i}$ of $\beta_{i}$ by $\sigma$, i.e., we extend the domain set of $\boldsymbol{\sigma}_{-i} \in \boldsymbol{\Sigma}_{-i}$ to $\boldsymbol{\Sigma}$ by formally including also $\Sigma_{i}$.

Then the Nash equilibrium definition $\hat{\sigma}$ corresponds mathematically to the definition of a fixed point of the joint best-response function $\boldsymbol{\beta}$, i.e. $\hat{\boldsymbol{\sigma}}=\boldsymbol{\beta}(\hat{\boldsymbol{\sigma}})$. Thus we replace the search for Nash equilibrium in our power control model and proof instead the existence of a fixed point $\hat{\boldsymbol{\sigma}}$ of $\boldsymbol{\beta}$.

To continue, note that if sets $\Sigma_{i}$ are non-empty, compact and convex sets for all $i \in \mathscr{I}$, then also Cartesian products 
$\boldsymbol{\Sigma}$ and $\boldsymbol{\Sigma}_{-i}$ of such sets are non-empty, compact and convex sets. At this stage, we can readily use the Brouwer's fixed point theorem as a special case of the Kakutani's theorem [17] and claim the existence of a Nash equilibrium fixed point in power control with general continuous $\boldsymbol{\beta}$, taking advantage of the fact that these assign $\sigma_{i}$ to $\sigma_{-i}$, which can be formally rewritten as theorem's mapping of $\boldsymbol{\Sigma}$ into $\boldsymbol{\Sigma}$.

Although practical devices are using discrete memory look-up tables with $\Delta \neq 0$, all our theoretical results for continuous best-response functions are still applicable with a near-optimal performance if the values of functions $\beta_{i}$ are quantization in the memory look-up table with a suitably small step-size $\Delta$.

Such quasi-continuity is important for the above result. First of all, if $\boldsymbol{\beta}$ exhibited very large step-size $\Delta$, the power control might be oscillating around the equilibrium with large amplitudes. More importantly however, discontinuous best-response functions might not theoretically admit Nash equilibrium $\hat{\boldsymbol{\sigma}}=\boldsymbol{\beta}(\hat{\boldsymbol{\sigma}})$, i.e., have no intersection of $\boldsymbol{\beta}(\hat{\boldsymbol{\sigma}})$ with line $\hat{\boldsymbol{\sigma}}$. This means that is not possible to prove the general existence of Nash equilibria for discontinuous or only piecewise continuous best-response functions. Thus $\Delta$ must be kept as small as possible.

\section{Step 2: How to Assure Convergence to Equilibria}

Having shown the way of assuring the existence of Nash equilibria in our power control schemes, we now focus on assuring their convergence to such equilibria. In order to grasp this problem mathematically and consequently also on a practical level, we first formally define the power control dynamics of our proposed schemes.

Assume a state variable $\boldsymbol{\theta}$ as a vector of all transmit powers $\sigma_{i}$ in the network at time $t$ in order to capture the dynamic evolution of network power conditions. Then we can model the power control dynamics of our game by a continuous-time dynamics of $\boldsymbol{\theta}$, which is governed by a standard law of motion, given by $N$ coupled first-order scalar differential equations [18], [19]

$$
\dot{\boldsymbol{\theta}}(t)=\boldsymbol{\beta}(\boldsymbol{\theta}(t))
$$

where $\dot{\boldsymbol{\theta}}$ denotes the derivative of $\boldsymbol{\theta}$ with respect to time $t$.

Defining now the convergence of the power control dynamics (1), we will for analytical purposes conveniently refer to the general convergence of the best-response dynamics (1) to the equilibrium point $\hat{\boldsymbol{\theta}}$ as to the "stability" of this point (see [15] for details). We say the equilibrium $\hat{\boldsymbol{\theta}}$ is

- stable if for each $\epsilon>0$, there is $\delta=f(\epsilon)>0$ such that $\|\boldsymbol{\theta}(0)\|<\delta$ implies $\|\boldsymbol{\theta}(t)\|<\epsilon$ for all $t \geq 0$;

- asymptotically stable if it is stable and $\delta$ can be chosen such that $\|\boldsymbol{\theta}(0)\|<\delta$ implies $\lim _{t \rightarrow \infty} \boldsymbol{\theta}(t)=\mathbf{0}$; and

- unstable if it is not stable.

According to this definition, the convergence of power control dynamics of $\boldsymbol{\theta}$ (i.e. the stability of $\hat{\boldsymbol{\theta}}$ ) implies that $\boldsymbol{\theta}$ remains after some time in a close neighborhood of $\hat{\boldsymbol{\theta}}$, called the region of stability, whose size is arbitrarily given by the function $f$. Importantly, $\boldsymbol{\theta}$ can also oscillate around the equilibrium $\hat{\boldsymbol{\theta}}$ with acceptably small amplitudes (see [14] for illustrations). In the case of asymptotical stability, the close neighborhood is moreover shrinking in time and reduces to the equilibrium point itself as time goes to infinity. Similarly, instability of the equilibrium is equivalent to the divergence of the power control process.

The above three types of stability can be illustrated by a simple pendulum example. A pendulum has two equilibrium positions. The first one is given by the position, in which the pendulum is standing upside down on the suspension point. This equilibrium state is unstable as any minor spatial shift or deviation leads to the pendulum's overturn to a second equilibrium state - the usual downward oriented position. This second position is in contrary stable, because when neglecting friction the pendulum oscillates around the equilibrium if deflected. Assuming friction, the stability of the downward position is then asymptotical, because the oscillation amplitude decreases in time due to energy dissipation. In analogical terms, the pendulum converges (asymptotically) to its (asymptotically) stable bottom equilibrium and diverges from the unstable top equilibrium.

Having formalized the notion of convergence of the power control dynamics, we can now state a design technique, which guarantees the convergence of the designed power control in the sense of the above definitions. This technique is defined in such a way so that it is valid for both proposed algorithms, yet in the case of the second one, instead of $\beta_{i}$, a substitute functions $\beta_{i}^{\prime}=\beta_{i}-\sigma_{i}$ should be used with no loss of generality ${ }^{\dagger}$.

Design Technique 2a (Verifying power control convergence for given $\left.\beta_{i}\right)$ : The power dynamics of $\dot{\boldsymbol{\theta}}=\boldsymbol{\beta}(\boldsymbol{\theta}, t)$ from (1), where $\boldsymbol{\beta}(\mathbf{0}, t)=0$ for all $t$ is an equilibrium point (shifted with no loss of generality to origin) and $D \subset \mathfrak{R}^{N}$ is the domain containing the equilibrium $\boldsymbol{\theta}=\mathbf{0}$, converges to $\boldsymbol{\theta}$ if there exists a scalar function $V(\boldsymbol{\theta}, t): D \rightarrow \mathfrak{R}^{N}$ having continuous, first partial derivatives and satisfying

$$
\begin{aligned}
& V(\mathbf{0})=0 \text { and } V(\boldsymbol{\theta})>0 \text { in } D \backslash\{\mathbf{0}\} \text { and } \\
& \dot{V}(\boldsymbol{\theta}) \leq 0 \text { in } D
\end{aligned}
$$

In other words, the equilibrium point in origin is stable. Moreover, if $\dot{V}(\boldsymbol{\theta})<0$ in $D$, then the equilibrium point is asymptotically stable.

Proof: We build up on the results of [14] and [15]. To be able to predict future states (and thus also the convergence) of the power control dynamics of (1) for some initial $\boldsymbol{\theta}\left(t_{0}\right)$, there must exist a solution to (1). We understand by a solution of (1) over time interval $\left[t_{0}, t_{1}\right]$ with an initial value $\boldsymbol{\theta}_{0}$ a continuous function $\boldsymbol{\theta}(t):\left[t_{0}, t_{1}\right] \rightarrow \mathfrak{R}^{N}$ such that $\dot{\boldsymbol{\theta}}(t)$ is defined and $\dot{\boldsymbol{\theta}}(t)=\boldsymbol{\beta}(\boldsymbol{\theta}(t))$ for all $t \in\left[t_{0}, t_{1}\right]$.

In fact, the continuity assumption of $\boldsymbol{\beta}$ guarantees already the existence of at least one solution and as $\boldsymbol{\beta}$ is continuous in $\boldsymbol{\theta}$, the solution is also continuously differentiable (proof is omitted). However, as $\boldsymbol{\theta}$ is deterministic and timeinvariant over $\left[t_{0}, t_{1}\right]$, it is important to ensure the solution of

${ }^{\dagger}$ This holds for all design techniques in this section. 
(1) to be unique so that during exactly the same conditions said dynamics would lead always to the same state.

To ensure this we need all best-response functions to be also globally Lipschitz property in addition to their continuity [20]. We say that function $\boldsymbol{\beta}$ is Lipschitz, if $\boldsymbol{\beta}(\boldsymbol{\theta}, t)$ satisfies $|\boldsymbol{\beta}(\boldsymbol{\theta}, t)-\boldsymbol{\beta}(\boldsymbol{\tau}, t)| \leq L|\boldsymbol{\theta}-\boldsymbol{\tau}|$ for some positive constant $L$ and all $(\boldsymbol{\theta}, t)$ and $(\boldsymbol{\tau}, t)$ in some neighborhood of $\left(\boldsymbol{\theta}_{0}, t_{0}\right)$. Intuitively the Lipschitz condition means that a straight line joining two points of $\boldsymbol{\beta}$ cannot have slope with absolute value bigger than $L$. Clearly, this condition can be easily assumed to hold with respect to the quantized implementation of all best-response functions by a discrete memory look-up table.

It holds that under the above conditions, the unique solution continuously depends on the initial state $\boldsymbol{\theta}\left(t_{0}\right)$ [20], which gives us freedom to vary arbitrarily $\boldsymbol{\sigma}_{i}^{\text {init }}$. Then by Lyapunov indirect analysis the above theorem can be stated in accordance with [14].

We can see that the equilibrium is stable (resp. asymptotically stable), if there exists a continuously differentiable positive definite function $V(\boldsymbol{\theta})$ so that $\dot{V}(\boldsymbol{\theta})$ is negative semidefinite (resp. negative definite), and is unstable if $\dot{V}(\boldsymbol{\theta})$ is positive definite.

To give an illustrative analogy using the previous pendulum example, examining the energetic conditions of a pendulum is a possible analytical way of how to determine its stability. Representing the sum of the potential and kinetic energy of the pendulum as the function $V$, we can draw a conclusion on stability based on the analysis of its derivative $\dot{V}(\boldsymbol{\theta})$. Clearly, if $\dot{V}(\boldsymbol{\theta})=0$, the pendulum oscillates undisturbed and its energy remains constant. Energy dissipations due to friction imply a steady decrease of oscillations amplitude and therefore $\dot{V}(\boldsymbol{\theta})<0$. On the other hand, energy boosts increase the size of oscillations and correspond to $\dot{V}(\boldsymbol{\theta})>0$.

This being said, we proceed in answering how to design such a function $V$ so as to prove the convergence of the power control, defined by update functions $\beta_{i}$. A precise tutorial, consisting in the Schultz-Gibson variable gradient method [21], is given in the next design technique:

Design Technique $\mathbf{2 b}$ (Generating Lyapunov function $V$ for given $\beta_{i}$ ):

- Choose a candidate function $\operatorname{grad}(V)$ so that

$$
\dot{V}(\boldsymbol{\theta})=\operatorname{grad}(V)^{\mathrm{T}} \boldsymbol{\beta}=\frac{\partial V}{\partial \theta_{1}} \beta_{1}+\cdots+\frac{\partial V}{\partial \theta_{N}} \beta_{N}
$$

is negative definite, but leave some of its parameters undetermined.

- The only constraint on the choice of the candidate function is that its Jacobian matrix $\boldsymbol{J}$, defined as $\boldsymbol{J}=$ $\left[\frac{\partial \operatorname{grad}_{i}(V)}{\partial \theta_{j}}\right]$, must be symmetric, that is to say

$$
\frac{\partial \operatorname{grad}_{i}(V)}{\partial \theta_{j}}=\frac{\partial \operatorname{grad}_{j}(V)}{\partial \theta_{i}}
$$

- Calculate the function $V(\boldsymbol{\theta})$ by integration over the state space along the axes of $\mathfrak{R}^{N}$, whereby the undetermined parameters are specified to ensure the positive definiteness of the final $V(\boldsymbol{\theta})$.

Proof: From the equation $\dot{V}(\boldsymbol{\theta})=\operatorname{grad}(V)^{\mathrm{T}} \boldsymbol{\beta}=\frac{\partial V}{\partial \theta_{1}} \beta_{1}+$ $\cdots+\frac{\partial V}{\partial \theta_{N}} \beta_{N}$, we can see that $V$ can be found through integration of the gradient of $V$ over through state space from the origin to $\boldsymbol{\theta}$. Since $V(\boldsymbol{\theta})$ has to be scalar, the Jacobian matrix $\boldsymbol{J}$ must be symmetric for all $i, j=\mathscr{I}$. The corollary of this requirement is that the integration of $\dot{V}$ is independent on the integration path. So a useful calculus technique is to integrate along the axes of $\mathfrak{R}^{N}$ in order to simplify the integration process.

While the method is straightforward and general, it may not be always practical. In contrary, the following Krasovskii method based design technique quickly tests sufficient conditions for stability of nonlinear systems in the equilibrium neighborhood:

Design Technique 3 (Verifying power control convergence for given $\beta_{i}$ ): Assuming with no loss of generality a shift of the equilibrium point to the origin and defining Jacobian matrix as $\boldsymbol{J}=\left[\frac{\partial \beta_{i}}{\partial \theta_{j}}\right]_{\hat{\boldsymbol{\theta}}=\mathbf{0}}$ for all $i, j=\mathscr{I}$, the equilibrium is asymptotically stable, if all eigenvalues of $\boldsymbol{J}$ have real parts strictly negative.

Proof: This statement follows directly from [20].

Clearly, the system designer can just check the eigenvalues of $\boldsymbol{J}$. If all of them are smaller than one, then the equilibrium is stable. If however one eigenvalue has zero real part and the rest is having negative real parts, this method fails to determine the stability of the origin.

As we have seen, all the above design techniques help to establish local convergence of the power control in the equilibrium proximity. Yet as we mentioned already, a practical system designer is naturally interested in assuring a global asymptotical convergence to an equilibrium, i.e. the convergence for any initial transmit power. So the region of attraction $D$ must be clearly the whole strategy space $\boldsymbol{\Sigma}$.

Moreover, global convergence to one isolated equilibrium implies that there cannot exist by contradiction another isolated equilibrium. Thus, although the Design Technique 1 does not guarantee the equilibrium uniqueness (only its existence), if we assure global convergence to one isolated equilibrium, its uniqueness follows without assuming further unwanted restrictions or additional design steps. As such, unwanted oscillations between several equilibria can be elegantly avoided.

The following design technique states how to simply verify of a practical level, if the choice of $\beta_{i}$ allows for this. Such knowledge naturally completes the set of design techniques, necessary for assuring power control convergence.

Design Technique 4 (Verifying global convergence for given $\beta_{i}$ ):

If $V(\boldsymbol{\theta})>0$ from the previous design techniques is such that $\dot{V}(\boldsymbol{\theta})<0$ for all $\boldsymbol{\theta} \neq 0$ and approaches infinity for $\|\boldsymbol{\theta}\|$ increasing beyond bounds, i.e., $\|\boldsymbol{\theta}\| \rightarrow \infty \Rightarrow V(\boldsymbol{\theta}) \rightarrow \infty$, then the equilibrium point is globally asymptotically stable. 
Proof: For more details refer to [14] or [20].

\section{Design Example: Linear/Linearized Power Control}

In this section, we illustratively apply our previous results to the analysis of power control using linear or linearized nonlinear best-response functions in the form $\beta(\theta)=\boldsymbol{A} \theta+\boldsymbol{B}$ for some matrix $\boldsymbol{A}$ and vector $\boldsymbol{B}$. Of course, network designer can use this model for linearized analysis of nonlinear functions. In this case, we assume a continuous differentiability of $\boldsymbol{\beta}$ at equilibrium $\hat{\boldsymbol{\theta}}$ and a linearized approximation by $\boldsymbol{\beta}(\boldsymbol{\theta})=\boldsymbol{A} \boldsymbol{\theta}+\boldsymbol{B}$ with $\boldsymbol{A}=\frac{\partial \boldsymbol{\beta}(\hat{\boldsymbol{\theta}})}{\partial I}$ and $\boldsymbol{B}=\hat{\boldsymbol{\theta}}$.

In accordance with Design Technique 1, one can see that linear functions can be easily designed so as to assure the existence of Nash equilibrium, i.e., to make $\boldsymbol{A} \boldsymbol{\theta}+\boldsymbol{B}$ to map $\boldsymbol{\Sigma}$ to $\boldsymbol{\Sigma}$. Moreover, we can see that every row of $\boldsymbol{A}$ or $\boldsymbol{B}$ represents a different active link and $\boldsymbol{A}$ is therefore nonsingular, than there must exist only a unique solution to $\hat{\boldsymbol{\theta}}=\boldsymbol{A} \hat{\boldsymbol{\theta}}+\boldsymbol{B}$, representing coordinates of a unique isolated intersection point of all $\beta_{i}$. In other words, the Nash equilibrium of linear $\boldsymbol{\beta}$ is unique.

As discussed in Design Techniques $2 a$ and $2 b$, the designer can then verify the stability of the linear dynamics by finding a Lyapunov function $V(\boldsymbol{\theta})=\boldsymbol{\theta}^{\mathrm{T}} \boldsymbol{P} \boldsymbol{\theta}$, where $\boldsymbol{P}$ is the solution of Lyapunov equation $\boldsymbol{P} \boldsymbol{A}+\boldsymbol{A}^{\mathrm{T}} \boldsymbol{P}=-\boldsymbol{Q}$ for any positive definite symmetric $Q$ (see [15] for more details). Clearly, such Lyapunov functions prove not only stability, but also imply in accordance with Design Technique 4 asymptotical stability, because $\|\boldsymbol{\theta}\| \rightarrow \infty \Rightarrow \boldsymbol{\theta}^{\mathrm{T}} \boldsymbol{P} \boldsymbol{\theta} \rightarrow \infty$.

In [15], we also observe that the unique equilibrium point $\hat{\boldsymbol{\theta}}$ is globally asymptotically stable, if and only if all eigenvalues of $\boldsymbol{A}$ are smaller in absolute values than one. This observation implies that the linear power control dynamics stabilizes for any initial condition in the equilibrium with exponential motion $\boldsymbol{\theta}(t)=\boldsymbol{\theta}_{0} \exp (\boldsymbol{A} t)$, because the solutions of the system are exponential functions with exponents depending on eigenvalues of $\boldsymbol{A}$ (recall that any matrix $\boldsymbol{A}$ can be transformed by some nonsingular matrix into a diagonal matrix with elements corresponding to eigenvalues of $\boldsymbol{A}$, which are negative in this case).

\section{Simulations and Numerical Results}

\subsection{Illustration of Game-Theoretic Power Control}

To illustrate numerically the above described concepts and also to illustrate the favorable exponentially evolving convergence process of the linear or linearized power control dynamics to a unique equilibrium, we simulate similarly to [22] a simple ad hoc network with four links and normalized channel gains $\boldsymbol{H}^{\prime}(i j)=\left[h_{i j} / h_{i i}\right]$

$$
\boldsymbol{H}^{\prime}(i j)=\left[\begin{array}{llll}
1.00 & 0.12 & 1.63 & 0.42 \\
0.08 & 1.00 & 0.95 & 0.28 \\
0.36 & 3.33 & 1.00 & 1.51 \\
0.68 & 0.32 & 3.48 & 1.00
\end{array}\right],
$$

performing asynchronous power updates each $10 \mathrm{~ms}$. To show the adaptivity of the proposed algorithm, we will consider three admission events - (i) at time 0 links 1 and 2 simultaneously start to send continuous data flows; whereby (ii) links 3 and 4 enter the network at time $\mathrm{t}=100$ or $200 \mathrm{~ms}$ respectively; (iii) at time $400 \mathrm{~ms}$ link 1 ends its transmission.

We perform two power control simulations with this setup. First, we use a linear best-response algorithm of the first proposed type for ergodic channels (Fig. 2)

$$
B_{i}\left(\boldsymbol{h}_{-i}^{\mathrm{T}} \boldsymbol{\sigma}_{-i}\right)=h_{i i} P_{i}^{\max }-\alpha_{i} \boldsymbol{h}_{-i}^{\mathrm{T}} \boldsymbol{\sigma}_{-i}
$$

where $P_{i}^{\max }=1 \mathrm{~W}$ represents network maximum transmit power limit. Coefficients $\alpha_{i} \in[6.7 ; 2.66 ; 4.47 ; 7.1]$ were to set to mutually different random numbers in order to illustrate numerically different preferences on best-response functions among individual network links. Their values are in accordance with above design techniques. Then we perform a comparative simulation based on power control algorithm derived from [8], which is using the following transmit power update rule

$$
\begin{aligned}
& \sigma_{i}^{k+1}=\min \left[\operatorname{argmax}_{\sigma_{i}^{k} \in \Sigma_{\mathrm{i}}} u\left(\sigma_{i}^{k}, \boldsymbol{\sigma}_{-i}^{k}\right)\right] \\
& u=8\left(1-2 \exp \left(\frac{-h_{i i} \sigma_{i}^{k}}{2 \boldsymbol{h}_{-i}^{\mathrm{T}} \boldsymbol{\sigma}_{-i}^{k}}\right)\right)^{80} / \sigma_{i}^{k}-\sigma_{i}^{k}
\end{aligned}
$$

Left part of Fig. 4 shows the resulting evolution of transmit power in time for all transmitters and both algorithms (3) and (4). We clearly observe the impact of all three admission events, given by a sudden activation of links 3 and 4 or retreat of link 4, which require both algorithms to adaptively redefine power conditions in the network. In either case, algorithms properly react by converging within about $80 \mathrm{~ms}$ to a newly defined Nash equilibrium, in which according to definition no link is changing its power anymore

Linear Best-Response Scheme for Ergodic Channels
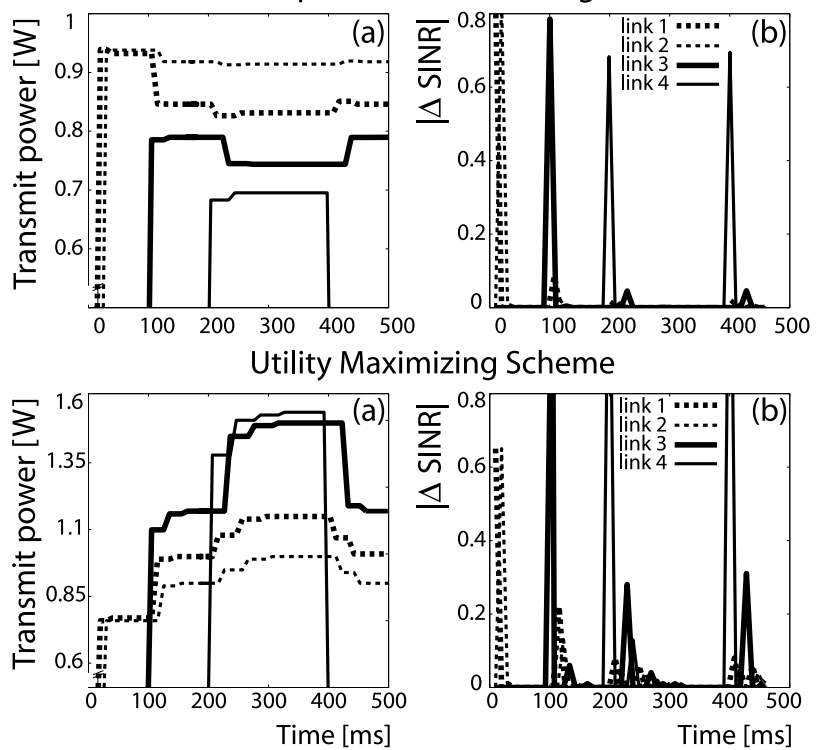

Fig. 4 Evolution of (a) transmit powers and (b) corresponding SINR increments (absolute value) for linear best-response scheme and utility based scheme of [8] with admission events at time $100,200,400 \mathrm{~ms}$. 


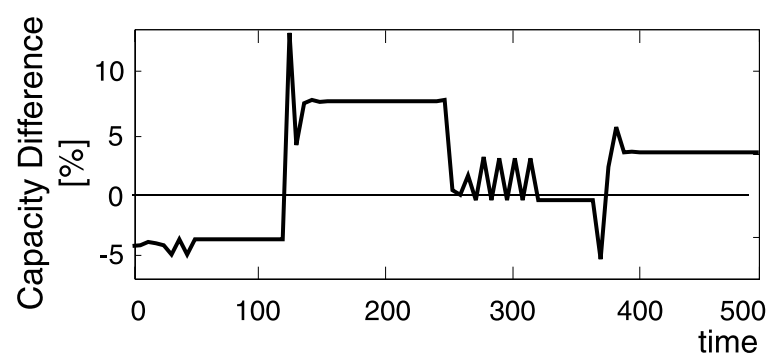

Fig. 5 Proportional difference of total network Shannon AWGN channel capacity between linear best-response scheme and utility maximizing scheme from [8].

(flat parts of graph lines).

Note however that the linear algorithm converges significantly faster than the utility maximizing one of [8]. This fact can be clearly observed in the right part of Fig. 4, showing in absolute values the increments of individual links' SINRs between consecutive time samples. Non-zero SINR increments represent the process of convergence to a Nash equilibrium, whereby its achievement corresponds to flat zero-valued parts of plot lines.

To evaluate the energetic efficiency in terms of achievable throughput, parameters of both algorithms were set so that they perform about the same in terms of total Shannon AWGN channel capacity (differing in only $4.8 \%$ as shown in Fig. 5). Yet the total power expenditure of the proposed algorithm is $24.3 \%$ smaller than the one of the compared algorithm [8], thus the proposed algorithm is energetically more efficient.

\subsection{Performance Analysis}

In the next, we are interested in how can our concept of allocating transmit power, based solely on experienced local co-channel interference, perform in terms of corresponding SINRs. This is an important issue, because generally speaking the above work does not concern directly some form of target SINR allocation as e.g. in [22].

Testing the performance by evaluating the allocated SINRs is however reasonable as many fundamental formulas of the wireless engineering theory such as link's modulation bit-error rate, robustness of coding schemes, receiver properties, equalization efficiency, channel capacity etc. depend on the relative ratio between its allocated transmit power and experienced interference [3] and not on allocated absolute transmit power values.

As we have seen in Sect. 6, the favorable existence of a unique Nash equilibrium and exponential speed of convergence is theoretically no directly linked to the particular value of best-response function coefficients $\alpha_{i}$. Therefore it remains to show in more detail that the proposed approach to power control has the potential to provide for a satisfactory power allocation, which we will prove by the following general simulation.

Its setup is based on a square area of $10 \mathrm{~km} \times 10 \mathrm{~km}$ with changing number of links from 1 to 100 . All links

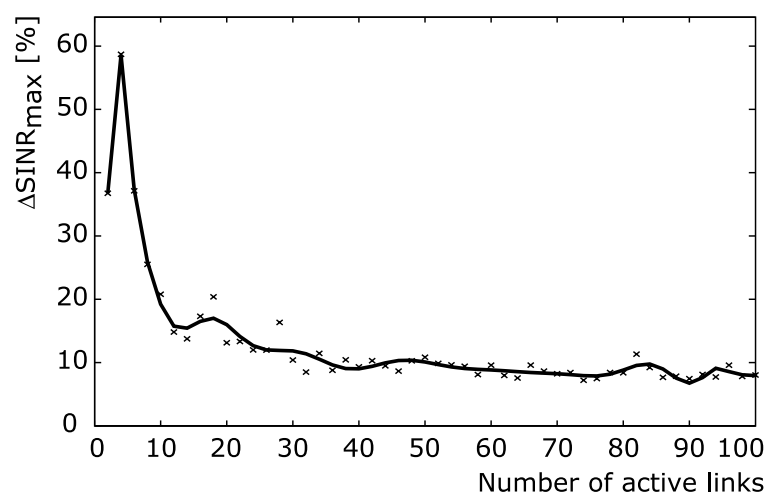

Fig. 6 Improvement of the average network SINR by the proposed algorithm with respect to the comparison one, shown as a function of increasing number of active network links. Data are averaged over $10^{4}$ topologies and interpolated with a polynomial of 16-th order.

are distributed in the network area uniformly distributively and use linear best-response functions from (3) with $\alpha_{i}=1$. Channel gains $h_{i j}$ are given by path loss with exponent 3.5.

We compare SINRs allocated by this linear bestresponse algorithm with SINRs of a comparison algorithm, which allocates every link's transmit power such that every receiver receives the same constant power. This comparison algorithm can be viewed as inspired by [23]. To ensure a fair comparison, the actual value of said constant receiver power is given by the condition that the total sum of transmit powers used by the "constant power" algorithm is equal to the total sum of equilibrium powers of the linear bestresponse algorithm. The advantage of a comparison with this modern algorithm is that it allows us to conduct a fair comparison under precisely defined and still realistic conditions. A general comparison of our concept with some other known algorithms would not be very exact due to their fundamental differences to our concept and application specific constraints such as e.g. in [8].

During the simulation, we slowly increase the number of active and uniformly randomly distributed links in the network starting from 1 and ending by 100 and examine again, how the proposed algorithm can improve the link's SINRs compared to the comparison algorithm.

Figure 6 shows the achieved difference of average SINRs, allocated by both algorithms, as a function of increasing number of active network links. We can see that our proposed algorithm outperforms the constant received power algorithm in sparse networks by up to 60 percent and is still about 10 percent more efficient even in dense networks.

The large improvement difference between sparse and dense networks lies in the fact that in rather sparse networks the interference can be less homogenous and thus results in allocation of mutually more different powers with same best-response functions, giving more chances for a significant local SINR improvement, whereas in denser networks, the interference is more homogenous and yielding therefore comparable powers, yielding only lower and more balanced 
SINR improvement possibilities. Nevertheless, generally speaking it seems that in an ad hoc environment, the strategy to adaptively adjust transmit powers with respect to changing interference is more advantageous than the strategy of maintaining a constant received power.

\section{Conclusion}

In this paper, we proposed two novel schemes for distributed and asynchronous power control in modern wireless networks, using general best-response functions and a game-theoretical point of view. Restricting our model by only minimal possible mathematical assumptions, we assure global convergence to Nash equilibria and by stochastic approximation and time-averaging gain a robust performance in random channels of both ergodic and non-ergodic type. The presented work is entirely general and application independent. As an illustration of our results, we show that stable linear best-response power control converges with exponential growth to a unique Nash equilibrium and numerically outperforms comparable power control schemes.

\section{Acknowledgments}

This work is supported in part by the 21 st Century COE Program (Grant No. 14213201) and Grant-in-Aid for Scientific Research (A) (No. 16206040), both from the Japan Society for the Promotion of Science (JSPS).

\section{References}

[1] N. Bambos, "Toward power-sensitive network architectures in wireless communications: Concepts, issues, and design aspects," IEEE Pers. Commun. Mag., vol.5, no.3, pp.50-59, June 1998.

[2] "The 3rd generation partnership project (3GPP) website," 2007.

[3] J.G. Proakis, Digital Communications, Irwin/McGraw-Hill, 2001.

[4] J. Zander, "Performance of optimum transmitter power control in cellular radio systems," IEEE Trans. Veh. Technol., vol.41, no.1, pp.57-62, Feb. 1992.

[5] G. Foschini and Z. Miljanic, "A simple distributed autonomous power control algorithm and its convergence," IEEE Trans. Veh. Technol., vol.42, no.4, pp.641-646, Nov. 1993.

[6] S. Haykin, "Cognitive radio: Brain-empowered wireless communications,” IEEE J. Sel. Areas Commun., vol.23, no.2, pp.201-208, Feb. 2005.

[7] A. Goldsmith, Wireless Communications, Cambridge University Press, 2005.

[8] C.U. Saraydar, N.B. Mandayam, and D.J. Goodman, "Efficient power control via pricing in wireless data networks," IEEE Trans Commun., vol.50, no.2, pp.291-303, Feb. 2002.

[9] M. Hayajneh and C. Abdallah, "Distributed joint rate and power control game-theoretic algorithms for wireless data," IEEE Commun. Lett., vol.8, no.8, pp.511-513, Aug. 2004.

[10] L. Jing and Z. Jiliu, "Power control based on game theory and iterative multiuser detectors," Proc. International Conference on Communications, Circuits and Systems 2005, pp.458-461, Hong-Kong, May 2005

[11] F. Meshkati, M. Chiang, H. Poor, and S.C. Schwartz, "A gametheoretic approach to energy-efficient power control in multicarrier cdma systems," IEEE J. Sel. Areas Commun., vol.24, no.6, pp.11151129, June 2006.
[12] S. Kucera, S. Aïssa, and S. Yoshida, "General game-theoretic model for power control in wireless networks with multiple-access bands," submitted to IEEE Trans. Wireless Commun., 2007.

[13] S. Kucera, K. Yamamoto, and S. Yoshida, "A game-theoretic framework for distributed power control in wireless ad hoc networks,' Proc. IEEE PIMRC 2006, pp.1-5, Helsinky, Finland, Sept. 2006.

[14] S. Kucera, K. Yamamoto, and S. Yoshida, "Distributed power control for wireless ad hoc networks: A game-theoretic approach based on best-response functions," Proc. IEEE VTC Fall 2006, pp.1-5, Montreal, Canada, Sept. 2006.

[15] S. Kucera, S. Aïssa, K. Yamamoto, and S. Yoshida, "Asynchronous distributed power and rate control in ad hoc networks: A gametheoretic approach," submitted to IEEE Trans. Wirel. Commun., 2006.

[16] S. Verdú, Multiuser Detection, Cambridge University Press, New York, 1998.

[17] D. Fudenberg and J. Tirole, Game Theory, MIT Press, 1991.

[18] D. Fudenberg and D.K. Levine, The Theory of Learning in Games, MIT Press, 1999

[19] H.J. Kushner and G.G. Yin, Stochastic Approximation and Recursive Algorithms and Applications, Springer-Verlag New York, 2003.

[20] H.K. Khalil, Nonlinear Systems, Prentice Hall, 2002.

[21] D. Schultz and J.E. Gibson, "The variable gradient method for generating Lyapunov functions," AIEE Trans., vol.81, pp.203-209, 1962.

[22] T. Holliday, A. Goldsmith, P. Glynn, and N. Bambos, "Distributed power and admission control for time-varying wireless networks,' Proc. IEEE Globecom, pp.768-774, Dallas, Texas, Nov.-Dec. 2004

[23] W. Yu and J.M. Cioffi, "Constant-power waterfilling: Performance bound and low-complexity implementation," IEEE Trans. Commun., vol.54, no.1, pp.23-28, Jan. 2006.

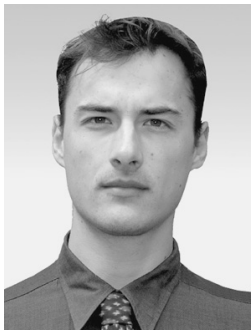

Stepan Kucera received the M.E. degree in communications and radio engineering in 2003 from Czech Technical University in Prague, Czech Republic. He is currently studying towards his Ph.D. degree at Graduate Schoo of Informatics at Kyoto University with the support of MEXT scholarship of Government of Japan. In 2006-2007, he was enrolled as a research assistant at the Center of Excellence for Research and Education of Fundamental Technologies at Kyoto University. He received the IEEE VTC Best Student Paper Award in 2006 and the IEEE Student Paper Award in 2007. His research interests concern wireless communications networks, in particular their game-theoretic modeling and analysis with respect to cross-layer optimization. He is a student member of IEEE.

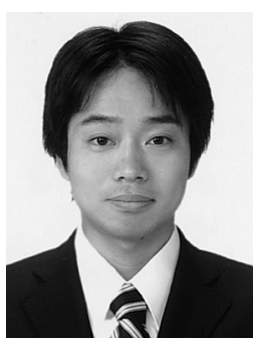

Koji Yamamoto received the B.E. degree in electrical and electronic engineering from Kyoto University in 2002, and the M.E. and Ph.D. degrees in informatics from Kyoto University in 2004 and 2005, respectively. Since 2005, he has been an assistant professor of the Graduate School of Informatics, Kyoto University. His research interests include multi-hop radio networks and distributed control. He received the PIMRC Best Student Paper Award in 2004 and the Ericsson Young Scientist Award in 2006. He

is a member of the IEEE. 


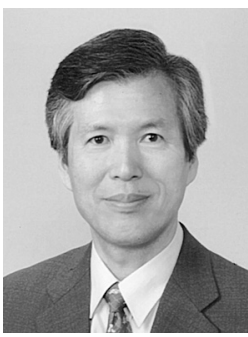

Susumu Yoshida received the B.E., M.E. and $\mathrm{Ph} . \mathrm{D}$. degrees in electrical engineering from Kyoto University, Kyoto, Japan in 1971, 1973 and 1978, respectively. Since 1973, he has been with the Faculty of Engineering, Kyoto University and currently he is a full professor of the Graduate School of Informatics, Kyoto University. During the last 30 years, he has been mainly engaged in the research of wireless personal communications. His current research interest includes highly spectrally efficient wireless transmission techniques and wireless ad hoc networks. During 19901991, he was a visiting scholar at WINLAB, Rutgers University, U.S.A. and Carleton University in Canada. He served as an Executive Committee Chair of PIMRC'99, Osaka and also as a Technical Program Committee Chair of IEEE VTC 2000-Spring, Tokyo. He was a guest editor of IEEE JSAC on Wireless Local Communications published in April and May 1996. He was a Director, Journal and Transactions of IEICE during 2002-2004, and is currently President-Elect of Communications Society of IEICE. He was awarded the Achievement Award in 1993 from the IEICE. 